\title{
Tuberculosis in Schools and Requirements for Prevention and Control in China
}

\author{
Hui Chen'; Yinyin Xia'; Canyou Zhang'; Hui Zhang'; Jun Cheng ${ }^{1, *}$; Yanlin Zhao ${ }^{1, *}$
}

In recent years, although tuberculosis (TB) control in schools has achieved success to some extent, TB outbreaks still occur in schools from time to time. There are gaps in the response to TB outbreaks, which should be identified and addressed through a series of integrated measures that should be implemented and further strengthened. This article summarizes the circumstances of TB outbreaks in schools, their characteristics, and experiences and lessons that can be learned to improve TB prevention and control.

\section{TUBERCULOSIS IN SCHOOLS}

In 2019, there were about 274 million students on campus (accounting for $19.6 \%$ of the national population) and 76 million newly enrolled students (1). The prevalence of $\mathrm{TB}$ in schools has been declining overall but has shown a slight rise in recent years. The reported incidence of TB among students dropped from 20.64/100,000 enrolled students in 2010 to $13.39 / 100,000$ in 2015 but increased to $17.50 / 100,000$ in 2019. During the same period, the percentage of student TB cases in all TB cases dropped from $4.94 \%$ to $3.97 \%$ and then rose to $6.19 \%(2-5)$. More than $100 \mathrm{~TB}$ outbreaks in schools have been reported since 2008; the number hit a peak in 2017 and then declined year over year. TB outbreaks often occurred in boarding schools with $70 \%$ being senior high schools or technical secondary schools and about $20 \%$ being private schools.

All TB outbreak investigation reports during 2017-2019 were collected from the Emergency Public Health Events Reporting System of China CDC and were analyzed for common characteristics. Overall, 48 TB outbreaks in schools were reported from 2017 to 2019, 36 in senior high schools, 8 in colleges and universities, 2 in junior high schools, and 2 in vocational schools. More types of schools were found reporting TB outbreaks in these 3 years, being different from that before 2017 . The proportion of outbreaks in senior high schools dropped from $77 \%$ in 2016 to
$50 \%$ in 2019 , and the $\mathrm{TB}$ outbreaks in vocational schools were reported. On average, 24 patients were found in each outbreak, and there were 9 outbreaks that had more than 30 patients found. There were 2 public health emergencies caused by the spread of multidrug-resistant TB (MDR-TB) in schools.

\section{EXPERIENCE AND LESSONS FOR TB CONTROL IN SCHOOLS}

Experiences and lessons were summarized from these TB outbreaks. First, the routine measurements for TB prevention and control in schools were inadequate. Students did not receive TB checks at the time of enrollment. Within the 48 schools with TB outbreaks, 16 schools failed to perform any physical examinations for newly enrolled students or excluded TB checks from physical examinations; 4 schools performed TB tests exclusively using chest X-ray or using $\mathrm{TB}$ antibody screening, which breached the guidelines; at least 17 schools failed to implement morning checkups for TB symptoms or any tracing and registration for students with illness, leading to the failure of early detection of students with TB symptoms or suspected TB patients. Classrooms and dormitories in most schools were poorly ventilated; and 23 schools reported poor ventilation and no established regulation for ventilation.

Second, the diagnosis, treatment, and infectious case reporting at health facilities needed to be enhanced. The proportion of bacteriologically positive patients rose from $10 \%$ in 2017 to $23 \%$ in 2019 but remained very low. Underreporting for infectious disease cases has decreased, but there was a long interval of time between diagnosis and reporting, even longer than 2 weeks in some health facilities. These reports that did not follow professional guidelines delayed the ability for schools to promptly respond to TB cases.

Third, the outbreak response measures at lower-level CDCs have yet to be standardized. The timeliness of close contact screening has improved: the median 
number of days of the interval between the diagnosis of index cases and close contacts screening has dropped from 7 days to 3 days from 2017 to 2019. Detailed investigation and analysis should be conducted scientifically to determine the reasonable scope of contact screening. However, the scope of screening was often insufficient in the early stage of responses. In addition, the screening methods needed to be further standardized. In some outbreak responses, symptom screening was the only method carried out, chest fluoroscopy was performed instead of chest X-ray, or tuberculin skin test (TST) was not performed.

Finally, there was a lack of scientific understanding of TB among students and parents, and delays in seeking health care were prevalent. The average interval between the onset of symptoms and the diagnosis at the designated health facility was more than 70 days. For fear of being stigmatized against or negatively impacting their studies, some students and their parents choose to conceal the illness and continue school attendance, leading to continuous transmission in schools.

\section{REQUIREMENTS FOR TB CONTROL IN SCHOOLS}

In recent years, health departments and education departments have attached great importance to $\mathrm{TB}$ control in schools, striving to raise $\mathrm{TB}$ detection rates and reduce TB outbreaks in schools. However, there are still many shortcomings in TB control work, which should be recognized, and a series of integrated measures should be implemented and further strengthened (๑):

First, schools should be instructed on TB control. Through work meetings and training, schools should be guided to carry out physical examinations with all newly enrolled students, and the frequency of TB screening should be elevated in some areas and schools when necessary (7-8). Morning check-ups and tracing of students with illnesses should be reinforced, and the referral and arrival rates of students with suspected symptoms and suspected patients should be raised. In addition, the environment in schools should be improved, especially for ventilation in classrooms and dormitories.

Second, all areas should establish effective channels for communication among schools, local CDCs, and health facilities within their jurisdictions to facilitate information exchange and standardize case reporting and registration. Capacities in surveillance and early warning systems should be strengthened with enhanced efficiency of data utilization. Early warning information based on individual cases should be thoroughly explored (9), and automated-alert methods and thresholds should be optimized to identify highrisk schools promptly (10).

Third, when a TB outbreak occurs, the principle of simultaneous investigation, management, and improvement should be followed to identify the cause of the outbreak and control and prevent further spread of TB. Once any TB cases are detected in a school, an investigation should be carried out as soon as possible to assess and judge the situation and the possibility of TB spread. All cases should be searched and verified case by case. The time distribution of the cases, case distribution in classes and dormitories, distribution of population characteristics, and the correlation among them should be analyzed. Close contacts should be screened for TB: symptom screening, TST, and chest X-ray should be performed simultaneously for those aged 15 and above; for those under 15 years of age, symptom screening and TST should be performed first, and chest X-ray should then be performed for those being found with suspected symptoms or strong positive TST. According to the situation on the spot and the findings of screening, the scope of screening should be expanded as appropriate. In general, screening should first be performed with teachers and students who were in the same class or dormitory as the patient. If a new TB case is found, screening must be extended to students and teachers on the same teaching building floor and dormitory floor as the case. In addition, it should be noted that family members in close contact with TB cases should also be screened. All active TB cases should be incorporated into the scope of TB control programs for case management and standardized treatment. Those who meet the criteria for suspension of schooling must suspend their schooling for medical treatment. Suspected cases should be isolated from other students before a final diagnosis is made. On the basis of excluding TB and related contraindications, it was recommended that students with strong positive TST alone receive preventive treatment intervention with their informed consent.

Finally, TB health education in schools should be improved in a variety of ways. The awareness of TB identification and prevention among students and their parents should be enhanced. Sound healthcare practices and moral literacy should be developed, 
including prompt health-seeking upon illness, disclosure of disease diagnosis without concealment, and voluntary suspension of school attendance when necessary.

doi: $10.46234 / \mathrm{ccdcw} 2021.005$

\# Corresponding authors: Jun Cheng, chengjun@chinacdc.cn; Yanlin Zhao, zhaoyl@chinacdc.cn.

${ }^{1}$ Chinese Center for Diseases Control and Prevention, Beijing, China.

Submitted: May 26, 2020; Accepted: December 30, 2020

\section{REFERENCES}

1. National Bureau of Statistics of the People's Republic of China. China statistical yearbook. Beijing: China Statistics Press. 2020. http://www. stats.gov.cn/tjsj/ndsj/2020/indexch.htm. (In Chinese).

2. Du X, Chen W, Huang F, Wang W, Yan FX, Wang HF, et al. Characteristics analysis of national student reported incidence of tuberculosis, 2004-2008. Chin J Health Educ 2009;25(11):803 - 6, 810. http://d.wanfangdata.com.cn/periodical/ChlQZXJpb2RpY2FsQ0h JTmV3UzIwMjAxMjI4Eg96Z2prankyMDA5MTEwMDEaCDl2d2F mMmg1. (In Chinese).

3. Xia YY, Ma Y, Chen W, Cheng SM. Epidemic features of pulmonary tuberculosis in students in China: data mining based on national disease surveillance system. Chin J Antituberculosis 2013;35(11): 871 - 5. http:// d.wanfangdata.com.cn/periodical/zgflzz201311005. (In Chinese).

4. Chen W, Chen QL, Xia YY, Cheng SM. Analysis of the characteristics of national TB epidemic situation in schools from 2008 to 2012. Chin J Antituberculosis 2013;35(12):948 - 54. http://d.wanfangdata.com. $\mathrm{cn} /$ periodical/zgflzz201312001. (In Chinese).

5. Chen H, Xia YY, Zhang CY, Cheng J, Zhang H. Epidemic trends and characteristics of pulmonary tuberculosis in students in China from 2014 to 2018. Chin J Antituberculosis 2019;41(6):662 - 8. http://dx.doi.org/10.3969/j.issn.1000-6621.2019.06.01. (In Chinese).

6. National Health and Family Planning Commission of the People's Republic of China, Ministry of Education of the People's Republic of China. National norm of tuberculosis prevention and control in school (2017). 2017. http://www.moe.gov.cn/srcsite/A17/moe_943/s3285/ 201707/t20170727_310182.html. (In Chinese).

7. Pang Y, Wu CG, Liu Y, Zhang T, Duan WX, Yu Y, et al. Disposal measures of school tuberculosis outbreak in Chongqing. China Trop Med 2019;19(1):97 - 9. http://dx.doi.org/10.13604/j.cnki.46-1064/r. 2019.01.25. (In Chinese).

8. Xiao X, Chen J, Li XQ, Xia Z, Luan RR, Rao LX, et al. Analysis of the epidemiological characteristics of tuberculosis students in Shanghai from 2009 to 2017. Chin J Antituberculosis 2020;42(5):498 - 502. http://dx.doi.org/10.3969/j.issn.1000-6621.2020.05.015. (In Chinese).

9. Chen YZ, Tan SY. Research progress of strategy on tuberculosis aggregation epidemic monitoring and early warning in school. J Tuber Lung Health 2015;4(1):57 - 60. http://dx.doi.org/10.3969/j.issn.20953755.2015.01.013. (In Chinese).

10. Cheng J, Liu JJ. Current status and progress of surveillance and automated-alert for tuberculosis in school. Chin J Antituberculosis 2020;42(5):436 - 41. http://dx.doi.org/10.3969/j.issn.1000-6621.2020. 05.005. (In Chinese). 